\title{
Orthogonal bases of Hermitean monogenic polynomials: an explicit construction in complex dimension 2
}

\author{
F. Brackx*, H. De Schepper*, R. Lávička ${ }^{\dagger}$ and V. Souček ${ }^{\dagger}$ \\ ${ }^{*}$ Clifford Research Group, Department of Mathematics, Faculty of Engineering, Ghent University, Belgium \\ ${ }^{\dagger}$ Mathematical Institute, Charles University, Sokolovská 83, 18675 Praha 8, Czech Republic
}

\begin{abstract}
In this contribution we construct an orthogonal basis of Hermitean monogenic polynomials for the specific case of two complex variables. The approach combines group representation theory, see [5], with a Fischer decomposition for the kernels of each of the considered Dirac operators, see [4], and a Cauchy-Kovalevskaya extension principle, see [3].
\end{abstract}

Keywords: Hermitean Clifford analysis, orthogonal basis PACS: 02.30 .-f (MSC 30G35)

\section{BASICS OF HERMITEAN CLIFFORD ANALYSIS}

Let $\left(e_{1}, \ldots, e_{m}\right)$ be an orthonormal basis of $\mathbb{R}^{m}$, then multiplication in the complex Clifford algebra $\mathbb{C}_{m}$ is governed by the rule $e_{\alpha} e_{\beta}+e_{\beta} e_{\alpha}=-2 \delta_{\alpha \beta}, \alpha, \beta=1, \ldots, m$, whence $\mathbb{C}_{m}$ is generated additively by the elements $e_{A}=e_{j_{1}} \ldots e_{j_{h}}$, where $A=\left\{j_{1}, \ldots, j_{h}\right\} \subset\{1, \ldots, m\}$, with $1 \leq j_{1}<j_{2}<\cdots<j_{h} \leq m$, and $e_{\emptyset}=1$.

The framework for Hermitean Clifford analysis is introduced by means of a complex structure, i.e. an $\mathrm{SO}(m ; \mathbb{R})-$ element $J$ with $J^{2}=-\mathbf{1}$ (see [1, 2]). So, the dimension is forced to be even: $m=2 n$. Usually $J$ is chosen to act upon the generators of $\mathbb{C}_{2 n}$ as $J\left[e_{j}\right]=-e_{n+j}$ and $J\left[e_{n+j}\right]=e_{j}, j=1, \ldots, n$. By means of the projection operators $\pm \frac{1}{2}(\mathbf{1} \pm i J)$ associated to $J$, first the Witt basis elements $\left(\mathfrak{f}_{j}, f_{j}^{\dagger}\right)_{j=1}^{n}$ for $\mathbb{C}_{2 n}$ are obtained: $\mathfrak{f}_{j}=\frac{1}{2}(\mathbf{1}+i J)\left[e_{j}\right]=\frac{1}{2}\left(e_{j}-i e_{n+j}\right)$ and $\mathfrak{f}_{j}^{\dagger}=-\frac{1}{2}(\mathbf{1}-i J)\left[e_{j}\right]=-\frac{1}{2}\left(e_{j}+i e_{n+j}\right), j=1, \ldots, n$, satisfying the relations $\mathfrak{f}_{j} \mathfrak{f}_{k}+\mathfrak{f}_{k} \mathfrak{f}_{j}=\mathfrak{f}_{j}^{\dagger} \mathfrak{f}_{k}^{\dagger}+\mathfrak{f}_{k}^{\dagger} \mathfrak{f}_{j}^{\dagger}=0$ and $\mathfrak{f}_{j} \mathfrak{f}_{k}^{\dagger}+\mathfrak{f}_{k}^{\dagger} \mathfrak{f}_{j}=\delta_{j k}, j, k=1, \ldots, n$. Next, a vector $\left(x_{1}, \ldots, x_{n}, y_{1}, \ldots, y_{n}\right) \in \mathbb{R}^{2 n}$ is identified with $\underline{X}=\sum_{j=1}^{n}\left(e_{j} x_{j}+e_{n+j} y_{j}\right)$, producing the Hermitean variables $\underline{z}=\frac{1}{2}(\mathbf{1}+i J)[\underline{X}]=\sum_{j=1}^{n} \mathfrak{f}_{j} z_{j}$ and $\underline{z}^{\dagger}=-\frac{1}{2}(\mathbf{1}-i J)[\underline{X}]=\sum_{j=1}^{n} f_{j}^{\dagger} \bar{z}_{j}$, expressed in the complex variables $z_{j}=x_{j}+i y_{j}$ and their conjugates $\bar{z}_{j}=x_{j}-i y_{j}, j=1, \ldots, n$. Finally, the Dirac operator $\partial_{\underline{X}}=\sum_{j=1}^{n}\left(e_{j} \partial_{x_{j}}+e_{n+j} \partial_{y_{j}}\right)$ gives rise to the Hermitean Dirac operators $\partial_{\underline{z}}^{\dagger}=\frac{1}{4}(\mathbf{1}+i J)\left[\partial_{\underline{X}}\right]=\sum_{j=1}^{n} \mathfrak{f}_{j} \partial_{\bar{z}_{j}}$ and $\partial_{\underline{z}}=$ $-\frac{1}{4}(\mathbf{1}-i J)\left[\partial_{\underline{X}}\right]=\sum_{j=1}^{n} f_{j}^{\dagger} \partial_{z_{j}}$, involving the Cauchy-Riemann operators $\partial_{\bar{z}_{j}}=\frac{1}{2}\left(\partial_{x_{j}}+i \partial_{y_{j}}\right)$ and their conjugates $\partial_{z_{j}}=\frac{1}{2}\left(\partial_{x_{j}}-i \partial_{y_{j}}\right), j=1, \ldots, n$. The Hermitean variables and Dirac operators are isotropic, whence the Laplacian decomposes as $\Delta_{2 n}=4\left(\partial_{\underline{z}} \partial_{\underline{z}}^{\dagger}+\partial_{\underline{z}}^{\dagger} \partial_{\underline{z}}\right)$, while also $\underline{z} \underline{\dagger}^{\dagger}+\underline{z^{\dagger}} \underline{z}=|\underline{z}|^{2}$.

We take functions with values in an irreducible representation $\mathbb{S}_{n}$ of $\mathbb{C}_{2 n}$, called spinor space, which is realized within $\mathbb{C}_{2 n}$ using a primitive idempotent $I=I_{1} \ldots I_{n}$, with $I_{j}=\mathfrak{f}_{j} \mathrm{f}_{j}^{\dagger}, j=1, \ldots, n$. With that choice $\mathbb{S}_{n} \equiv \mathbb{C}_{2 n} I \cong \bigwedge_{n}^{\dagger} I$, where $\bigwedge_{n}^{\dagger}$ denotes the Grassmann algebra generated by the $\mathfrak{f}_{j}^{\dagger}$ s, since $\mathfrak{f}_{j} I=0$. Hence $\mathbb{S}_{n}$ decomposes into homogeneous parts as $\mathbb{S}_{n}=\bigoplus_{r=0}^{n} \mathbb{S}_{n}^{(r)}=\bigoplus_{r=0}^{n}\left(\bigwedge_{n}^{\dagger}\right)^{(r)} I$, with $\left(\bigwedge_{n}^{\dagger}\right)^{(r)}=\operatorname{span}_{\mathbb{C}}\left(\mathfrak{f}_{k_{1}}^{\dagger} \wedge \mathfrak{f}_{k_{2}}^{\dagger} \wedge \cdots \wedge \mathfrak{f}_{k_{r}}^{\dagger}:\left\{k_{1}, \ldots, k_{r}\right\} \subset\{1, \ldots, n\}\right)$.

A continuously differentiable function $g$ in an open region $\Omega$ of $\mathbb{R}^{2 n}$, taking values in $\mathbb{S}_{n}$, then is called (left) Hermitean monogenic in $\Omega$ iff it satisfies in $\Omega$ the system $\partial_{\underline{z}} g=0=\partial_{z}^{\dagger} g$. A major difference with Euclidean Clifford analysis concerns the underlying group invariance. Where $\partial_{\underline{X}}$ is invariant under the action of $\mathrm{SO}(m)$, the system invariance of $\left(\partial_{\underline{z}}, \partial_{\underline{z}}^{\dagger}\right)$ breaks down to the group $\mathrm{U}(n)$, see e.g. [1, 2]. For this reason $\mathrm{U}(n)$ will play a fundamental role in the construction of an orthogonal basis of Hermitean monogenic polynomials, as explained in [5].

The spaces of homogeneous polynomials on $\mathbb{C}^{n}$ with bidegree of homogeneity $(a, b)$ in $\left(\underline{z}, \underline{z}^{\dagger}\right)$, taking values in $\mathbb{S}_{n}^{(r)}$, will be denoted by $\mathscr{P}_{a, b}^{r}\left(\mathbb{C}^{n}\right)$. By $\mathscr{M}_{a, b}\left(\mathbb{C}^{n}\right)$ we denote the space of Hermitean monogenic polynomials of bidegree $(a, b)$ in $\left(\underline{z}, \underline{z}^{\dagger}\right)$, and by $\mathscr{M}_{a, b}^{r}\left(\mathbb{C}^{n}\right)$ its subspace with values in $\mathbb{S}_{n}^{(r)}$; the latter may be further split as

$$
\mathbb{S}_{n}^{(r)} \equiv\left(\bigwedge_{n}^{\dagger}\right)^{(r)} I=\left(\bigwedge_{n-1}^{\dagger}\right)^{(r)}\left(\mathfrak{f}_{1}^{\dagger}, \ldots, \mathfrak{f}_{n-1}^{\dagger}\right) I \bigoplus\left(\bigwedge_{n-1}^{\dagger}\right)^{(r-1)}\left(\mathfrak{f}_{1}^{\dagger}, \ldots, \mathfrak{f}_{n-1}^{\dagger}\right) \mathfrak{f}_{n}^{\dagger} I
$$


whence we can decompose polynomials in $\mathscr{M}_{a, b}^{r}\left(\mathbb{C}^{n}\right)$ as $p_{a, b}=p_{a, b}^{0} I+p_{a, b}^{1} f_{n}^{\dagger} I$, with $p_{a, b}^{0}$ taking values in $\left(\bigwedge_{n-1}^{\dagger}\right)^{(r)}\left(\mathfrak{f}_{1}^{\dagger}, \ldots, f_{n-1}^{\dagger}\right)$ and $p_{a, b}^{1}$ taking values in $\left(\bigwedge_{n-1}^{\dagger}\right)^{(r-1)}\left(\mathfrak{f}_{1}^{\dagger}, \ldots, f_{n-1}^{\dagger}\right)$. Note that for $r=0$ or $r=n$ one of these components becomes trivial. In the same order of ideas we single out the variables $\left(z_{n}, \bar{z}_{n}\right)$ and rewrite the Hermitean variables as $\underline{z}=\underline{\tilde{z}}+\mathfrak{f}_{n} z_{n}$ and $\underline{z}^{\dagger}=\underline{\tilde{z}}^{\dagger}+\mathfrak{f}_{n}^{\dagger} \bar{z}_{n}$, and the Hermitean Dirac operators as $\partial_{\underline{z}}=\widetilde{\partial}_{\underline{z}}+\mathfrak{f}_{n}^{\dagger} \partial_{z_{n}}$ and $\partial_{z}^{\dagger}=\widetilde{\partial}_{z}^{\dagger}+\mathfrak{f}_{n} \partial_{\bar{z}_{n}}$. We will consider restrictions to $\left\{z_{n}=0=\bar{z}_{n}\right\}$, identified with $\mathbb{C}^{n-1}$. The following results were then proven in [3].

Proposition 1. (i) Given the polynomial $p_{a, b-j}^{0} I \in \operatorname{Ker}\left(\widetilde{\partial}_{\underline{z}}\right)$ on $\mathbb{C}^{n-1}(j=0, \ldots, b)$, there exists a unique polynomial $M_{a, b, j}^{0} \in \mathscr{M}_{a, b}\left(\mathbb{C}^{n}\right)$, given by

$$
M_{a, b, j}^{0}=\bar{z}_{n}^{j}\left(\sum_{k=0}^{\min (2 a+1,2(b-j))} \frac{1}{\left\lfloor\frac{k}{2}\right\rfloor !} \frac{1}{\left\lfloor\frac{k+1}{2}\right\rfloor !}\left(z_{n} \widetilde{\partial}_{\underline{z}} \mathfrak{f}_{n}+\bar{z}_{n} \widetilde{\partial}_{\underline{z}}^{\dagger} f_{n}^{\dagger}\right)^{k} p_{a, b-j}^{0} I\right)
$$

such that $\left.\partial_{\bar{z}_{n}}^{j} M_{a, b, j}^{0}\right|_{\mathbb{C}^{n-1}}=p_{a, b-j}^{0}$ I and all other derivatives w.r.t. $\bar{z}_{n}$ vanish in $\mathbb{C}^{n-1}$.

(ii) Given the polynomial $p_{a-i, b}^{1} f_{n}^{\dagger} I \in \operatorname{Ker}\left(\widetilde{\partial}_{\underline{z}}^{\dagger}\right)$ on $\mathbb{C}^{n-1}(i=0, \ldots, a)$, there exists a unique polynomial $M_{a, b, i}^{1} \in$ $\mathscr{M}_{a, b}\left(\mathbb{C}^{n}\right)$, given by

$$
M_{a, b, i}^{1}=z_{n}{ }^{i}\left(\sum_{k=0}^{\min (2 a, 2 b+1)} \frac{1}{\left\lfloor\frac{k}{2}\right\rfloor !} \frac{1}{\left\lfloor\frac{k+1}{2}\right\rfloor !}\left(z_{n} \widetilde{\partial}_{\underline{z}} \mathfrak{f}_{n}+\bar{z}_{n} \widetilde{\partial}_{\underline{z}}^{\dagger} \mathfrak{f}_{n}^{\dagger}\right)^{k} p_{a-i, b}^{1} \mathfrak{f}_{n}^{\dagger} I\right)
$$

such that $\left.\partial_{z_{n}}^{i} M_{a, b, i}^{1}\right|_{\mathbb{C}^{n-1}}=p_{a-i, b}^{1} \mathfrak{f}_{n}^{\dagger} I$ and all other derivatives w.r.t. $z_{n}$ vanish in $\mathbb{C}^{n-1}$.

The polynomial $M_{a, b, j}^{0}$ (respectively $M_{a, b, i}^{1}$ ) is called the Hermitean Cauchy-Kovalevskaya extension of the initial polynomial $p_{a, b-j}^{0} I$ (respectively the initial polynomial $\left.p_{a-i, b}^{1} \mathfrak{f}_{n} I\right)$. This CK extension will play an important role in the construction of the desired orthogonal basis. Indeed, introducing, as in [5], the following spaces of initial polynomials:

$$
\begin{aligned}
\mathscr{A}_{a, b-j}^{r} & =\left\{p_{a-i, b}^{0} I \mid p_{a-i, b}^{0} I \in \operatorname{Ker}\left(\widetilde{\partial}_{\underline{z}}\right) \cap \mathscr{P}_{a, b-j}^{r}\left(\mathbb{C}^{n-1}\right)\right\} \\
\mathscr{B}_{a-i, b}^{r} & =\left\{p_{a-i, b}^{1} f_{n}^{\dagger} I \mid p_{a-i, b}^{1} I \in \operatorname{Ker}\left(\partial_{\underline{z}}^{\dagger}\right) \cap \mathscr{P}_{a-i, b}^{r-1}\left(\mathbb{C}^{n-1}\right)\right\}
\end{aligned}
$$

the CK extension map is an isomorphism from $\oplus_{j=0}^{b} \mathscr{A}_{a, b-j}^{r} \oplus \oplus_{i=0}^{a} \mathscr{B}_{a-i, b}^{r}$ to $\mathscr{M}_{a, b}^{r}$, commuting with the action of $\mathrm{U}(n-1)$, whence it yields a splitting of $\mathscr{M}_{a, b}^{r}$ into a direct sum of $\mathrm{U}(n-1)$ invariant subspaces. Since the initial polynomials on $\mathbb{C}^{n-1}$ for the CK extension have to be submit to the compatibility condition of being either in the kernel of $\widetilde{\partial}_{\underline{z}}$ or in the kernel of $\widetilde{\partial}_{\underline{z}}^{\dagger}$, the so-called Fischer decomposition of these kernels in terms of Hermitean monogenics will also be involved. Under the action of $\mathrm{U}(n-1)$, see [4], the space $\operatorname{Ker}_{a, b}^{r}\left(\widetilde{\partial}_{\underline{z}}\right) \equiv \operatorname{Ker}\left(\widetilde{\partial}_{\underline{z}}\right) \cap \mathscr{P}_{a, b}^{r}\left(\mathbb{C}^{n-1}\right)$ has the multiplicity free irreducible decomposition

$$
\operatorname{Ker}_{a, b}^{r}\left(\widetilde{\partial_{\underline{z}}}\right)=\mathscr{M}_{a, b}^{r} \bigoplus_{j=0}^{\min (a, b-1)}|\underline{z}|^{2 j} \underline{z}^{\dagger} \mathscr{M}_{a-j, b-j-1}^{r-1} \bigoplus_{j=0}^{\min (a-1, b-1)} \mid \underline{z^{2 j}}\left(\underline{z^{\dagger}} \underline{z}+\frac{(a-j-1+r)}{(a+r)} \underline{z} \underline{z}^{\dagger}\right) \mathscr{M}_{a-j-1, b-j-1}^{r}
$$

and the space $\operatorname{Ker}_{a, b}^{r-1}\left(\widetilde{\partial}_{\underline{z}}^{\dagger}\right) \equiv \operatorname{Ker}\left(\widetilde{\partial}_{\underline{z}}^{\dagger}\right) \cap \mathscr{P}_{a, b}^{r-1}\left(\mathbb{C}^{n-1}\right)$ has the multiplicity free irreducible decomposition

$$
\operatorname{Ker}_{a, b}^{r-1}\left(\widetilde{\partial}_{\underline{z}}^{\dagger}\right)=\mathscr{M}_{a, b}^{r-1} \bigoplus_{j=0}^{\min (a-1, b)}|\underline{z}|^{2 j} \underline{z} \mathscr{M}_{a-j-1, b-j}^{r} \bigoplus_{j=0}^{\min (a-1, b-1)}|\underline{z}|^{2 j}\left(\underline{z} \underline{z}^{\dagger}+\frac{(b-j-1+n-r+1)}{(b+n-r+!)} \underline{z} \underline{z}^{\dagger}\right) \mathscr{M}_{a-j-1, b-j-1}^{r-1}
$$

It now becomes clear that, once the desired bases have been constructed in dimension $n-1$, these results can been used as building blocks in the above Fischer decompositions, yielding bases for the spaces $\mathscr{A}_{a, b-j}^{r}$ and $\mathscr{B}_{a-i, b}^{r}$ of initial polynomials. Subsequent application of the CK extension procedure, will then produce a basis for the space $\mathscr{M}_{a, b}^{r}$ in dimension $n$, which, by construction, will be orthogonal w.r.t. any $\mathrm{U}(n)$ invariant inner product.

We will now follow this general procedure as explained above, and, in more detail, in [5], to explicitly obtain orthogonal bases for the spaces $\mathscr{M}_{a, b}^{r}\left(\mathbb{C}^{2}\right), r=0,1,2,(a, b) \in \mathbb{N}^{2}$. Since the procedure is inductive, we need however to start with the case $n=1$. 


\section{THE CASE $n=1$}

In this case we are considering polynomials $f\left(z_{1}, \bar{z}_{1}\right)$ defined in the complex plane and taking values in the spinor space $\mathbb{S}_{1}=\operatorname{span}_{\mathbb{C}}\left\{1, \mathfrak{f}_{1}^{\dagger}\right\} I$. The Hermitean Dirac operators are simply $\widetilde{\partial}_{\underline{z}}=\mathfrak{f}_{1}^{\dagger} \partial_{z_{1}}$ and $\widetilde{\partial}_{\underline{z}}^{\dagger}=\mathfrak{f}_{1} \partial_{\bar{z}_{1}}$, whence Hermitean monogenicity means nothing else but anti-holomorphy in the case $r \stackrel{\underline{z}}{=} 0$ and holomorphy in the case $r=1$. The symmetry group here is $\mathrm{U}(1) \simeq \mathrm{SO}(2)$.

For $r=0$ the $\mathrm{U}(1)$ modules $\widetilde{\mathscr{M}}_{0, b}^{0}$ are given by $\operatorname{span}\left\{\frac{\bar{z}_{1}^{b}}{b !} I\right\}, b=0,1,2, \ldots$. They have highest weight $(-b)$.

For $r=1$ the $\mathrm{U}(1)$ modules $\widetilde{\mathscr{M}_{a, 0}^{1}}$ are given by $\operatorname{span}\left\{\frac{z_{1}^{a}}{a !} f_{1}^{\dagger} I\right\}, a=0,1,2, \ldots$. They have highest weight $(a+1)$.

\section{THE CASE $n=2$}

Now we consider polynomials $f\left(z_{1}, \bar{z}_{1}, z_{2}, \bar{z}_{2}\right)$ taking values in the spinor space $\mathbb{S}_{2}=\operatorname{span}_{\mathbb{C}}\left\{1, f_{1}^{\dagger}, f_{2}^{\dagger}, f_{1}^{\dagger} f_{2}^{\dagger}\right\} I$. If $r=0$ or $r=2$ we are again confronted with (anti-)holomorphy, see [2], so we will focus on the interesting case $r=1$.

The dimension of the U(2) module $\mathscr{M}_{a, b}^{1}$ is $a+b+2$, see [3]. Each of the spaces of initial polynomials $\mathscr{A}_{a, b-j}^{1}$, $j=0, \ldots, b$ and $\mathscr{B}_{a-i, b}^{1}, i=0, \ldots, a$, is one-dimensional. The general theory of the CK extension procedure, see [3], predicts that the compatibility conditions imposed on these initial polynomials will be trivially fulfilled, so they simply are all homogeneous polynomials in the variables $z_{1}$ ans $\bar{z}_{1}$ of the appropriate bidegree, which is moreover confirmed by the Fischer decompositions (1)-(2):

$$
\begin{array}{lll}
\mathscr{A}_{a, b-j}^{1}=\operatorname{span}_{\mathbb{C}}\left\{(-1)^{b-j} \frac{z_{1}^{a}}{a !} \frac{\bar{z}_{1}^{b-j}}{(b-j) !} \mathfrak{f}_{1}^{\dagger} I\right\}, & j=0, \ldots, b \\
\mathscr{B}_{a-i, b}^{1}=\operatorname{span}_{\mathbb{C}}\left\{(-1)^{b} \frac{z_{1}^{a-i}}{(a-i) !} \frac{\bar{z}_{1}^{b}}{b !} \mathfrak{f}_{2}^{\dagger} I\right\}, & i=0, \ldots, a
\end{array}
$$

By CK extension each of the spaces of initial polynomials thus gives rise to exactly one Hermitean monogenic basis polynomial, together yielding an orthogonal basis for $\mathscr{M}_{a, b}^{1}$, see [5]. These basis polynomials are respectively given by

$$
\begin{aligned}
M_{a, b, j}^{0}= & \sum_{k=0}^{\min (a, b-j)}(-1)^{b-j-k} \frac{z_{2}^{k}}{k !} \frac{\bar{z}_{2}{ }^{k+j}}{(k+j) !} \frac{z_{1}{ }^{a-k}}{(a-k) !} \frac{\bar{z}_{1}{ }^{b-j-k}}{(b-j-k) !} \mathfrak{f}_{1}^{\dagger} I \\
& +\sum_{k=0}^{\min (a, b-j-1)}(-1)^{b-j-k-1} \frac{z_{2}{ }^{k}}{k !} \frac{\bar{z}_{2}{ }^{k+j+1}}{(k+j+1) !} \frac{z_{1}{ }^{a-k}}{(a-k) !} \frac{\bar{z}_{1}^{b-j-k-1}}{(b-j-k-1) !} \mathfrak{f}_{2}^{\dagger} I, \quad j=0, \ldots, b \\
M_{a, b, i}^{1}= & \sum_{k=0}^{\min (a-i, b)}(-1)^{b-k} \frac{z_{2}{ }^{k+i}}{(k+i) !} \frac{\bar{z}_{2}^{k}}{k !} \frac{z_{1}{ }^{a-i-k}}{(a-i-k) !} \frac{\bar{z}_{1}{ }^{b-k}}{(b-k) !} \mathfrak{f}_{2}^{\dagger} I \\
& +\sum_{k=0}^{\min (a-i-1, b)}(-1)^{b-k} \frac{z_{2}{ }^{k+i+1}}{(k+i+1) !} \frac{\bar{z}_{2}{ }^{k}}{k !} \frac{z_{1}{ }^{a-i-k-1}}{(a-i-k-1) !} \frac{\bar{z}_{1}{ }^{b-k}}{(b-k) !} \mathfrak{f}_{1}^{\dagger} I, \quad i=0, \ldots, a
\end{aligned}
$$

The following properties may then be verified right away.

Property 1. Under derivation with respect to the "new" variables $\left(z_{2}, \bar{z}_{2}\right)$, the orthogonal basis polynomials of $\mathscr{M}_{a, b}^{1}$ act as follows:

$$
\begin{array}{lll}
\partial_{z_{2}} M_{a, b, i}^{1}=M_{a-1, b, i-1}^{1} & \partial_{\bar{z}_{2}} M_{a, b, i}^{1}=M_{a, b-1, i+1}^{1} & i=1, \ldots, a \\
\partial_{z_{2}} M_{a, b, j}^{0}=M_{a-1, b, j+1}^{0} & \partial_{\bar{z}_{2}} M_{a, b, j}^{0}=M_{a, b-1, j-1}^{0} & j=1, \ldots, b \\
\partial_{z_{2}} M_{a, b, 0}^{1}=M_{a-1, b, 0}^{0} & \partial_{\bar{z}_{2}} M_{a, b, 0}^{0}=M_{a, b-1,0}^{1} &
\end{array}
$$

Property 2. Under derivation with respect to the "old" variables $\left(z_{1}, \bar{z}_{1}\right)$, the orthogonal basis polynomials of $\mathscr{M}_{a, b}^{1}$ act as follows:

$$
\begin{aligned}
& \partial_{z_{1}} M_{a, b, i}^{1}=M_{a-1, b, i}^{1} \quad-\partial_{\bar{z}_{1}} M_{a, b, i}^{1}=M_{a, b-1, i}^{1} \quad i=0, \ldots, a \\
& \partial_{z_{1}} M_{a, b, j}^{0}=M_{a-1, b, j}^{0} \quad-\partial_{\bar{z}_{1}} M_{a, b, j}^{0}=M_{a, b-1, j}^{0} \quad j=0, \ldots, b
\end{aligned}
$$


Remark 1. Property 1 holds in any dimension n, whereas Property 2 is specific for the case $n=2$.

Remark 2. Since the orthogonal Hermitean monogenic basis polynomials are determined only up to a constant, the final expressions may always be normalized, according to some preferred behaviour or property. Here, we have in fact normalized all initial data by requiring that

(i) if $p_{a, b}^{0} I \in \mathscr{A}_{a, b}^{1}$, then $\partial_{z_{1}}^{a}\left(-\partial_{\bar{z}_{1}}\right)^{b}\left[p_{a, b}^{0} I\right]=\mathfrak{f}_{1}^{\dagger} I$;

(ii) if $p_{a, b}^{1} \mathfrak{f}_{2}^{\dagger} I \in \mathscr{B}_{a, b}^{1}$, then $\partial_{z_{1}}^{a}\left(-\partial_{\bar{z}_{1}}\right)^{b}\left[p_{a, b}^{1} \mathfrak{f}_{2}^{\dagger} I\right]=\mathfrak{f}_{2}^{\dagger} I$.

These normalization conditions are reflected in the eventual orthogonal basis as follows:

$$
\partial_{z_{1}}^{a}\left(-\partial_{\bar{z}_{1}}\right)^{b-j}\left[M_{a, b, j}^{0}\right]=\frac{\bar{z}_{2}^{j}}{j !} f_{1}^{\dagger} I, \quad \partial_{z_{1}}^{a-i}\left(-\partial_{\bar{z}_{1}}\right)^{b}\left[M_{a, b, i}^{1}\right]=\frac{z_{2}^{i}}{i !} f_{2}^{\dagger} I
$$

Finally let us check the branching rules for the space $\mathscr{M}_{a, b}^{1}$ with highest weight $\lambda=(a+1,-b)$. From group representation theory, see e.g. [6], we know that when restricting the symmetry to U(1), the irreducible U(2) module $\mathscr{M}_{a, b}^{1}$ decomposes into irreducible U(1) modules as

$$
\mathscr{M}_{a, b}^{1}=\bigoplus_{\mu \succ \lambda} V_{\mu}=\bigoplus_{k=-b}^{a+1} V_{k}
$$

where each summand appears with multiplicity one; this decomposition is orthogonal w.r.t. any U(2) invariant scalar product on $\mathscr{M}_{a, b}^{1}$. On the other hand the Fischer decompositions (1)-(2) produce the U(1) irreducible components of the spaces of initial data $\mathscr{A}_{a, b-j}^{1}(j=0, \ldots, b)$ and $\mathscr{B}_{a-i, b}^{1}(i=0, \ldots, a)$. Assuming that $a>b$ (the cases $a \leq b$ being similar) we have in fact that $\mathscr{A}_{a, b-j}^{1}$ is a shifted version of the U(1) module $\widetilde{\mathscr{M}}_{a-b+j, 0}^{1}$ with highest weight $(a-b+j+1)$, for all $j=0, \ldots, b$. Similarly, $\mathscr{B}_{a-i, b}^{1}$ is a shifted version of the $\mathrm{U}(1)$ module $\widetilde{\mathscr{M}}_{a-i-b-1,0}^{1}$ with highest weight $(a-i-b)$, for all $i=0, \ldots, a-b-1$, while $\mathscr{B}_{a-i, b}^{1}$ is a shifted version of $\widetilde{\mathscr{M}}_{0, b-a+i}^{0}$ with highest weight $(a-i-b)$, for all $i=a-b, \ldots, a$. As the CK extension map is an isomorphism between the initial data space $\oplus_{j=0}^{b} \mathscr{A}_{a, b-j}^{1} \oplus \oplus_{i=0}^{a} \mathscr{B}_{a-i, b}^{1}$ and the space $\mathscr{M}_{a, b}^{1}$, which commutes with the action of $\mathrm{U}(1)$, our construction of the orthogonal basis of $\mathscr{M}_{a, b}^{1}$ exactly yields the above splitting of $\mathscr{M}_{a, b}^{1}$ into the direct sum of $a+b+2 \mathrm{U}(1)$ invariant subspaces $V_{k}, k=-b, \ldots, a+1$.

Remark 3. For completeness we mention here the cases $r=0$ and $r=2$. For $r=0$ the orthogonal basis of $\mathscr{M}_{0, b}^{0}$ consists of all homogeneous anti-holomorphic polynomials in $\left(\bar{z}_{1}, \bar{z}_{2}\right)$, i.e. $\frac{\bar{z}_{1}{ }^{b-j}(b-j) !}{\bar{z}_{2} j}, j=0, \ldots, b$, while for $r=2$, the orthogonal basis of $\mathscr{M}_{a, 0}^{2}$ consists of all homogeneous holomorphic polynomials in $\left(z_{1}, z_{2}\right)$, i.e. $\frac{z_{1}^{a-i}}{(a-i) !} \frac{z_{2}^{i}}{i !}, i=0, \ldots, a$.

\section{ACKNOWLEDGMENTS}

R. Lávička and V. Souček acknowledge support by grant MSM 0021620839 and by grant GA CR 201/08/0397.

\section{REFERENCES}

1. F. Brackx, J. Bureš, H. De Schepper, D. Eelbode, F. Sommen, V. Souček, Fundaments of Hermitean Clifford analysis - Part I: Complex structure, Compl. Anal. Oper. Theory 1(3) (2007), 341-365.

2. F. Brackx, J. Bureš, H. De Schepper, D. Eelbode, F. Sommen, V. Souček, Fundaments of Hermitean Clifford analysis - Part II: Splitting of $h$-monogenic equations, Complex Var. Elliptic Eq. 52(10-11) (2007), 1063-1079.

3. F. Brackx, H. De Schepper, R. Lávička, V. Souček, The Cauchy-Kovalevskaya extension theorem in Hermitean Clifford analysis (submitted).

4. F. Brackx, H. De Schepper, R. Lávička, V. Souček, Fischer decompositions of kernels of Hermitean Dirac operators. Submitted to: T.E. Simos, G. Psihoyios, Ch. Tsitouras, Numerical Analysis and Applied Mathematics, AIP Conference Proceedings, Rhodes, Greece (2010).

5. F. Brackx, H. De Schepper, R. Lávička, V. Souček, Gel'fand-Tsetlin procedure for the construction of orthogonal bases in Hermitean Clifford analysis. Submitted to: T.E. Simos, G. Psihoyios, Ch. Tsitouras, Numerical Analysis and Applied Mathematics, AIP Conference Proceedings, Rhodes, Greece (2010).

6. R. Goodman, N. Wallach: Representations and invariants of the classical groups, Cambridge University Press, 1998. 